that for $s=C, y^{\alpha}=$ const, $L$ being undefined. Hence in case (II) we are dealing with hyperspheres.

(III) $L^{2}+K=0$. In this case it has been demonstrated [2] that the vectors $N$ and hence the geodesic trajectories are all parallel. Consequently the family consists of limiting hypersurfaces or hyperhorospheres. They all have curvature zero.

Elliptic space admits of case (II) only, but the hyperspheres are geometrically the same as equidistant hypersurfaces.

\title{
REFERENCES
} 1949.

1. L. P. Eisenhart, Riemannian geometry, Princeton Univ. Press, Princeton, N. J.,

2. C. M. Fulton, Parallel vector fields, Proc. Amer. Math. Soc. 16 (1965), 136-137.

3. C. E. Weatherburn, An introduction to Riemannian geometry and the tensor calculus, University Press, Lambridge, 1957.

University of California, Davis

\section{THE RING $C(X)$ DETERMINES THE CATEGORY OF $X$}

W. W. COMFORT AND STELIOS NEGREPONTIS ${ }^{1}$

Summary and introduction. Our point of departure is the assertion (justified in Lemma 1.2) that certain topological properties of an arbitrary space $Y$ are inherited by each subset of $Y$ which meets each nonempty $G_{\delta}$ subset of $Y$. We consider specifically only the properties "is of second category" and "is a Baire space," but other properties in the same vein will occur readily to the reader. Specializing quickly to completely regular Hausdorff spaces, we recall that $X$ is $G_{\delta}$-dense (in the sense just described) in its Hewitt completion (= "realcompactification") $v X$, so that $X$ possesses each of the properties in question if and only if $v X$ does. This means that algebraic properties of the ring $C(X)$ of real-valued continuous functions on $X$ determine, for example, the category of $X$. (We make no claim that the conditions on $C(X)$ which determine whether or not $X$ is of second category are aesthetically pleasing or elegantly presented. For the record we give such criteria in 2.5 , but the merit of that section rests in its existence, not in its content.)

Since $G_{\delta}$-density is preserved by arbitrary products (in a sense

Presented to the Society, August 7, 1964; received by the editors July 6, 1964.

1 Each author received partial support from the National Science Foundation. 
made precise in 1.3), we see in 2.7 that a product of completely regular Hausdorff spaces $\prod_{\alpha \in A} X_{\alpha}$ enjoys either of the properties in question if and only if the product $\prod_{\alpha \in A}\left(v X_{\alpha}\right)$ does. A corollary is our final result, which can be deduced easily from Oxtoby's Theorem 6 in [3], but which we have not found stated explicitly in the literature: the product of pseudocompact spaces is a Baire space.

Definitions and theorms from the literature. A topological space is said to be of first category if it can be expressed as the countable union of subsets each of which is nowhere dense. Other topological spaces are of second category. Thus a space is of second category if and only if the countable intersection of dense, open subsets is nonempty. We say that a topological space is a Baire space if the countable intersection of dense, open sets is dense (equivalently: if each of its nonempty open subsets is, considered as a topological space, of second category).

The Hewitt space $v X$ associated with the completely regular Hausdorff space $X$ is that realcompact subset of the Stone-Cech compactification $\beta X$ to which each real-valued continuous function on $X$ admits a continuous extension. (For the definition of $\beta X$ and "realcompact," and for a careful proof of the existence and uniqueness of $v X$, we refer the reader to the Gillman-Jerison text [1]. In the interest of completeness, however, we include as Lemma 2.1 a result from [1].)

1. Category and Baire spaces. The straightforward proof of our first lemma is omitted.

1.1. Lemma. Let $X$ be dense in $Y$. If $X$ is of second category, then $Y$ is of second category. If $X$ is a Baire space, then $Y$ is a Baire space.

1.2. Lemma. Let $X$ be a subset of $Y$, and suppose that each nonempty $G_{\delta}$ subset of $Y$ meets $X$. If $Y$ is of second category, then $X$ is of second category. If $Y$ is a Baire space, than $X$ is a Baire space.

Proof. For the category proof, take $U_{0}=X$ in what follows; for the Baire space proof, let $U_{0}$ be an arbitrary nonempty open subset of $X$.

Given a sequence $\left\{U_{n}\right\}_{n=1}^{\infty}$ of dense open subsets of $X$, we are to show that $\bigcap_{n=1}^{\infty} U_{n}$ meets $U_{0}$. For $0 \leqq n<\infty$ there exists open $V_{n} \subset Y$ such that $V_{n} \cap X=U_{n}$ and $V_{n}$ is dense in $Y$ for $1 \leqq n<\infty$. The $G_{\delta}$ set $\bigcap_{n=0}^{\infty} V_{n}$, being nonempty, meets $X$. I.e., $\left(\bigcap_{n-1}^{\infty} U_{n}\right) \cap U_{0} \neq \varnothing$.

1.3. Lemma. Let $X=\prod_{\alpha \in A} X_{\alpha}$ and $Y=\prod_{\alpha \in A} Y_{\alpha}$, where for each $\alpha$ 
in the index set $A$ the set $X_{\alpha}$ is a subset of $Y_{\alpha}$ which meets each nonempty $G_{\delta}$ in $Y_{\alpha}$. Then $X$ meets each nonempty $G_{\delta}$ in $Y$.

Proof. Let $V=\bigcap_{n=1}^{\infty} V_{n} \neq \varnothing$, each of the open sets $V_{n}$ being of the form

$$
V_{n}=\prod_{\alpha \in A} V_{n, \alpha}
$$

where each $V_{n, \alpha}$ is an open subset of $Y_{\alpha}$ and for each $n$ the identity $V_{n, \alpha}=Y_{\alpha}$ holds for all but finitely many $\alpha$ in $A$.

Let $V_{\alpha}=\bigcap_{n=1}^{\infty} V_{n, \alpha}$. Then $V_{\alpha}$ is a nonempty $G_{\delta}$ in $Y_{\alpha}^{\prime}$, so there is a point $x_{\alpha}$ in $V_{\alpha} \cap X_{\alpha}$. Evidently that point of $Y$ whose $\alpha$ coordinate is $x_{\alpha}$ lies in $X \cap V$.

1.4. TheOREM. Let $X=\prod_{\alpha \in A} X_{\alpha}$ and $Y=\prod_{\alpha \in A} Y_{\alpha}$, where for each $\alpha$ in the index set $A$ the set $X_{\alpha}$ is a subset of $Y_{\alpha}$ which meets each nonempty $G_{\delta}$ in $Y_{\alpha}$. Then $X$ is of first category if and only if $Y$ is of first category, and $X$ is a Baire space if and only if $Y$ is a Baire space.

Proof. From 1.1, 1.2 and 1.3.

2. Products of realcompact spaces. In the preceding section we imposed no separation property on our spaces, but in what follows we shall consider only completely regular Hausdorff spaces.

A zero-set in $X$ is a set of the form $f^{-1}(0)$, where $f$ belongs to the ring $C(X)$ of all continuous real-valued functions on $X$. The space $X$ is said to be pseudocompact if each element of $C(X)$ has bounded range.

\subsection{Lemma. Each nonempty $G_{\delta}$ in $v X$ meets $X$.}

Proof. Since each nonempty $G_{\delta}$ contains a nonempty zero-set, it suffices to show that each nonempty zero-set in $v X$ meets $X$. Suppose, on the contrary, that $f \in C(v X)$ and $f(p)=0$ and $f^{-1}(0) \cap X=\varnothing$. The restriction of the function $1 / f$ to $X$ is continuous and real-valued on $X$, but it clearly admits no continuous extension to $p$, so $p \notin v X$.

2.2. Theorem. The space $X$ is of first category if and only if $v X$ is of first category, and $X$ is a Baire space if and only if $v X$ is a Baire space.

Proof. This follows from $1.1,1.2$ and 2.1.

2.3. Corollary. Let $X$ be dense in $Y$, and suppose that each function in $C(X)$ is the restriction to $X$ of some function in $C(Y)$. Then $X$ is of first category if and only if $Y$ is of first category, and $X$ is a Baire space if and only if $Y$ is a Baire space. 
Proof. We have $v X=v Y$, so the results follow from 2.2.

The following special case of Theorem 2.2 is included for amusement only.

2.4. COROLlARY. The realcompactification of a space of first category cannot be locally compact.

Proof. Any locally compact space is of second category.

The real maximal ideals of the ring $C(X)$ are by definition those maximal ideals $M$ for which the quotient field $C(X) / M$ is isomorphic to the real field. According to [1], a space $X$ is realcompact if and only if each real maximal ideal $M$ in $C(X)$ has the form

$$
M=\{f \in C(X): f(x)=0\}
$$

for some $x$ in $X$. The following determination of the category of $X$ by means of the ring $C(X)$ is valid because the topology on $X$ is the smallest making each member of $C(X)$ continuous. Of the two properties listed, the first says that each $\mathfrak{T}_{n}$ is closed, the second that the complement in $\mathfrak{T}(=v X)$ of each $\mathfrak{T}_{n}$ is dense.

2.5. TheOREM. In order that $X$ be of first category it is necessary and sufficient that the space TI of real maximal ideals in the ring $C(X)$ may be written in the form

$$
\mathfrak{T}=\bigcup_{n=1}^{\infty} \mathfrak{N}_{n}
$$

where for each integer $n$ we have

(i) for each $M$ in $\mathfrak{T} \backslash \mathfrak{T l}_{n}$ there exists $f$ in $C(X)$ such that $f \notin M$ and $f \in N$ whenever $N \in \Re_{n}$; and

(ii) if $f \in M$ whenever $M \in \mathfrak{M} \backslash \mathfrak{N}_{n}$, then $f$ is the zero element of $C(X)$.

2.6. Remark. The ring $C^{*}(X)$ of all bounded continuous realvalued functions on $X$ is clearly not sensitive enough to determine the category or the Baire-ness of $X$, since indeed $C^{*}(X)$ is ringisomorphic to $C^{*}(\beta X)$ and the compact Hausdorff space $\beta X$ is always a Baire space. The properties of $X$ here considered will of course be determined by $C^{*}(X)$ when $C^{*}(X)=C(X)$-i.e., when $X$ is pseudocompact-and it is exactly for such spaces $X$ that each nonempty $G_{\delta}$ in $\beta X$ meets $X$ (see Glicksberg [2], or exercise 6I of [1]). The fact, now obvious, that each pseudocompact space is a Baire space, is strengthened considerably in 2.8 below.

2.7. Theorem. Let $Y=\prod_{\alpha \in A} v X_{\alpha}$, and let $X=\prod_{\alpha \in A} X_{\alpha}$. Then $Y$ is of first category if and only if $X$ is of first category, and $Y$ is a Baire space if and only if $X$ is a Baire space. 
Proof. Each nonempty $G_{\delta}$ in $Y$ meets $X$ by 2.1 , so the result follows from 1.4 .

2.8. Corollary. The product of pseudocompact spaces is a Baire space.

Proof. This will follow from 2.7, once we have verified the familiar fact that $v Z=\beta Z$ whenever $Z$ is pseudocompact. To see this, notice that if $Z$ is pseudocompact then $\beta Z$ is a realcompact subset of $\beta Z$ to which each continuous real-valued function on $Z$ admits a continuous extension. Hence $\beta Z=v Z$.

2.9. Remarks. It is shown in [1] that the relation $v Z=\beta Z$ holds only when $Z$ is pseudocompact. The relation $v(X \times Y)=v X \times v Y$, which one of us will study in detail in a later communication, fails for many pairs $(X, Y)$; thus 2.7 above is substantially stronger than 2.2. Novák-Terasaka have given an example (recorded in 9.15 of [1]) of a pseudocompact space $G$ for which $G \times G$ is not pseudocompact; in this case $v(G \times G)$, which is not compact, is not even homeomorphic with the compact product space $v G \times v G$.

Oxtoby has given in [3] results which improve our Corollary 2.8 in several ways. We shall not copy from [3] his definition of "pseudocomplete," but we remark that each pseudocompact space is pseudocomplete. (To see this, choose each of Oxtoby's sets $B(n)$ to be the class of all nonempty open subsets of the pseudocompact space under study, and apply Lemma 9.13 of [1].) Our 2.8 now follows from the facts that the product of pseudo-complete spaces is pseudo-complete [3, Theorem 6], and that any pseudo-complete space is a Baire space [3, Remark (5.1)].

Oxtoby also includes in [3] an example of a (completely regular Hausdorff) Baire space $X$ such that $X \times X$ is of first category.

\section{REFERENCES}

1. Leonard Gillman and Meyer Jerison, Rings of continuous functions, Van Nostrand, Princeton, N. J., 1960.

2. Irving Glicksberg, Stone-Čech compactifications of products, Trans. Amer. Math. Soc. 90 (1959), 369-382. 166.

3. J. C. Oxtoby, Cartesian products of Baire spaces, Fund. Math. 49 (1961), 157-

The UNIVERSITy OF Rochester 